\title{
Kikuchi fujimoto disease mimicking lymphoma detected on pet-ct imaging in a pediatric patient
}

\begin{abstract}
Kikuchi-Fujimoto's disease (KFH), which is seen more often in young Asian women than in childhood, is a rare benign feature of PET-CT that can be confused with malignant lymphoma due to common cervical hypermetabolic lymphadenopathy and high SUV values. In this interesting case report, PET-CT imaging findings of a rare Kikuchi-Fujimoto disease mimicking lymphoma in a pediatric patient is presented.
\end{abstract}

\author{
Volume 5 Issue 4 - 2018 \\ Pelin Özcan Kara,' Zehra Pınar Koç, Elvan \\ Çağlar Çıtak, ${ }^{2}$ Feryal Karaman, ${ }^{3}$ Mehmet \\ Yaldız \\ 'Department of Nuclear Medicine, Mersin University, Turkey \\ ${ }^{2}$ Department of Pediatric Oncology, Mersin University, Turkey \\ ${ }^{3}$ Department of Pathology, Mersin University, Turkey
}

Correspondence: Pelin Ozcan Kara, Mersin University Hospital, Department of Nuclear Medicine, 33343 Mersin, Turkey, Tel, 903242410000 , Fax 903242410098 .

Email ppelinozcan@gmail.com

Received: March II, 2018| Published: July 05, 2018

\section{Introduction}

Kikuchi-Fujimoto's disease is a rare cause of cervical lymphadenopathy, also called subacute or histiocytic necrotizing lymphadenitis. Kikuchi-Fujimoto disease (KFD) is a benign and selflimited disorder. This disorder is initially described as a lymphadenitis with a focal proliferation of reticular cells. This disorder must be included in the differential diagnosis of 'lymph node enlargement' since its course and treatment differ dramatically from those of lymphoma, tuberculosis, and SLE. In this case report, FDG PETCT imaging findings of a rare Kikuchi-Fujimoto disease mimicking lymphoma in a pediatric patient is presented.

\section{Case report}

A 17-year-old male patient with a pre-diagnosed lymphoma who had hepatosplenomegaly on an abdominal US examination and lymphadenopathies thought to be compatible with malignancy

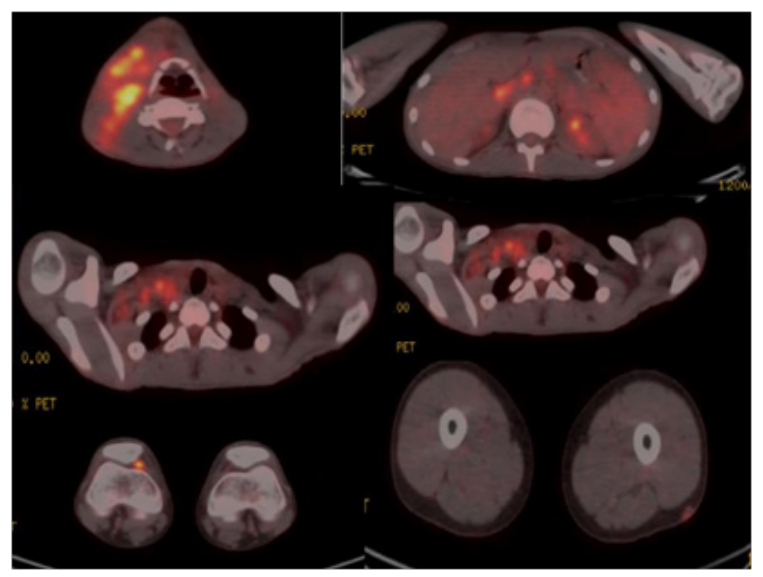

Figure I Axial PET-CT images show conglomerated hypermetabolic lymphadenopaties and relatively diffuse increased FDG uptake in the spleen. (Source:Authors). with heterogeneous enhancement at the level of the right neck of the neck in the US and CT examinations were undergone PET-CT imaging. PET-CT images from the calvarium to the soles of the foot revealed conglomerated lymphadenopathies (SUVmax: 4.9-11.1) on the right dominant bilateral cervical chain extending to the right supra-infraclavicular area. In addition, bilateral paraaortic, paraceliac, aortocaval and peripancreatic lymphadenopathies with a maximal size of $1 \mathrm{~cm}$ (SUVmax: 8.2) and diffuse FDG activity (SUVmax: 2.7/2.0) relatively increased compared to liver parenchyma in the spleen and minimal diffuse FDG activity in the bone marrow were detected. The left femur proximal posterolateral diaphyseal area showed a subcutaneous nodular probable inflammatory nodal low level of metabolic activity and possible pathological lymphadenopathy at the level of the right knee (SUVmax: 6.7) (Figure 1). A biopsy was suggested to the patient. Right cervical excisional lymph node biopsy was performed, lymphoma was excluded and the patient was diagnosed with Kikuchi Fujimoto Disease. Appropriate treatment was started and a few months later PET-CT was negative for all regions.

\section{Discussion}

Kikuchi-Fujimoto disease (KFD) is a benign and self-limited disorder, characterized by regional cervical lymphadenopathy. Initially described in Japan, KFD was first reported in 1972 almost simultaneously by Kikuchi ${ }^{1}$ ) and by Fujimoto et al., ${ }^{2}$ as a lymphadenitis with a focal proliferation of reticular cells accompanied by numerous histiocytes and extensive nuclear debris. ${ }^{3}$ This disorder must be included in the differential diagnosis of 'lymph node enlargement' since its course and treatment differ dramatically from those of lymphoma, tuberculosis, and SLE. The histological differential diagnosis of KFD mainly includes reactive lesions as lymphadenitis associated with SLE or herpes simplex, non-Hodgkin's lymphoma, plasmacytoid T-cell leukemia, Kawasaki's disease, myeloid tumor and even metastasic adenocarcinoma. ${ }^{4}$ Kikuchi-Fujimoto's disease $(\mathrm{KFH})$, which is seen more often in young Asian women than in childhood, is a rare benign feature of PET-CT that can be confused with malignant lymphoma due to common cervical hypermetabolic lymphadenopathy and high SUV values. Lymphoma is the third most common cancer in children. In a study by Tsujikawa $\mathrm{T}^{5}$ twenty-two patients with cervical lymphadenopathy ( 8 with KFD and 14 with 
NHL) underwent CT and FDG PET/CT scans to examine the cervical lymphadenopathy. As for SUV, a significant difference was observed only between indolent and aggressive (6.4 \pm 1.5 and 17.3 \pm 9.3 , $\mathrm{P}<0.05)$ NHL; however, KFD showed a significantly greater corSUV (23.8 \pm 10.6$)$ as compared with indolent NHL $(9.2 \pm 5.1, \mathrm{P}<0.05)$, which did not show a significant difference from aggressive NHL (21.4 \pm 10.2$)$. FDG PET/CT detected thoracoabdominal lesions in 2 patients $(25 \%)$ with KFD. KFD shows high FDG uptake for size, which may reflect the pathologic characteristics, including necrotizing lymphocytes and numerous histiocytes (macrophages) surrounding small necrotic foci. The authors concluded that FDG PET/CT will be useful for detecting noncervical lesions of KFD and distinguishing KFD from NHLs using both SUV and corSUV. In another study by Ji Eun Kim, ${ }^{6}$ the $18 \mathrm{~F}-\mathrm{FDG}$ $\mathrm{PET} / \mathrm{CT}$ maximum standardized uptake values (SUVmax) ranged from 8.3 to 22.5 (mean, 12.0) in KFDs, and from 5.8 to 34.3 (mean, 15.9 ) in MLs. There were no significant differences in SUVmax between KFDs and malignant lymphoma. In this study, patients with malignant lymphoma tended to present with extranodal involvement or huge conglomerated lymphadenopathies, in comparison with KFD patients who had generalized lymphadenopathy without extranodal involvement. Although, a number of differences have been reported in the literature, such as the absence of extranodal involvement sites in $\mathrm{KFH}$ and the absence of hepatosplenomegaly, relatively diffuse increased FDG uptake in the spleen and bone marrow has been noted, in this case and it is not possible to make a differential diagnosis with any of these parameters.

\section{Acknowledgements}

This research received no specific grant from any funding agency in the public, commercial, or not-for-profit sectors.

\section{Conflict of interest}

The author declares that there is no conflict of interest.

\section{References}

1. Kikuchi M. Lymphadenitis showing focal reticulum cell hyperplasia with nuclear debris and phagocytes: a clinicopathological study. Acta Hematol Jpn. 1972;35:379-380.

2. Fujimoto Y, Kozima Y, Yamaguchi K. Cervical subacute necrotizing lymphadenitis: a new clinicopathologic entity. Naika. 1972;20:920-927.

3. Dorfman RF. Histiocytic necrotizing lymphadenitis of Kikuchi and Fujimoto. Arch Pathol Lab Med. 1987;111(11):1026-1029.

4. Bosch X, Guilabert A, Miquel R, Campo E. Enigmatic Kikuchi-Fujimoto disease: a comprehensive review. Am J Clin Pathol. 2004; 122(1):141-152.

5. Tsujikawa $\mathrm{T}$, Tsuchida $\mathrm{T}$, Imamura $\mathrm{Y}$, et al. Kikuchi-Fujimoto disease: PET/CT assessment of a rare cause of cervical lymphadenopathy. Clin Nucl Med. 2011;36(8):661-664.

6. Ji Eun Kim, Eun Kyung Lee, Jae Min Lee, et al. Kikuchi-Fujimoto disease mimicking malignant lymphoma with 2-18[F]fluoro-2-deoxy-D-glucose PET/CT in children. Korean J Pediatr. 2014;57(5):226-231. 